\title{
Screening of Elevated Glucose Levels in Gingival Crevice Blood Using a Novel, Sensitive Self-Monitoring Device
}

\author{
H.-P. Müller E. Behbehani \\ Department of Surgical Sciences, Faculty of Dentistry, Kuwait University, Kuwait
}

\section{Key Words}

Periodontal disease · Diabetes mellitus · Gingival

crevice blood $\cdot$ Blood glucose $\cdot$ In-office testing

\begin{abstract}
Objective:To test the feasibility of using gingival crevice blood (GCB) collected during routine periodontal examination to estimate blood glucose levels using a novel and very sensitive self-monitoring device (FreeStyle ${ }^{\circledR}$ ). Subjects and Methods: Forty-six patients (20 male, 26 female; age range 12-56 years, mean age $36 \pm 11$ years) seeking dental treatment took part in the study. Three and 4 patients reported diabetes mellitus type 1 and 2, respectively; 24 had gingivitis, of which 22 were moderate or advanced periodontitis. Periodontal probing depth (PD) and clinical attachment loss was measured at 6 sites of every tooth present, and bleeding on probing (BOP) was recorded. A site with profuse BOP was chosen for glucose determination. Measurements in GCB were compared with those of conventional capillary fingerstick blood (CFB). Results: Sufficiently large GCB volumes provided glucometer readings in 32 cases (range 1.17-10.00 $\mathrm{mmol} / \mathrm{l}$ ). The CFB readings ranged between 1.39 and $11.50 \mathrm{mmol} / \mathrm{l}$. If low amounts of crevice blood prohibited a glucometer reading, significantly lower mean numbers of sites with BOP (26 vs. 67, p < 0.001) and elevated $P D \geq 4 \mathrm{~mm}$ ( 2 vs. $21, \mathrm{p}<0.05$ ) were seen.
\end{abstract}

Agreement between the two measurements was low, the mean difference was -1.22 , and limits of agreement (1.96 times the standard deviation of differences) were $\pm 2.89 \mathrm{mmol} / \mathrm{l}$. Conclusion: The present study failed to provide any evidence for the usefulness of GCB for testing blood glucose during routine periodontal examination.

Copyright $\odot 2004$ S. Karger AG, Basel

\section{Introduction}

Diabetes mellitus (DM) is one of the most important risk factors for destructive periodontitis. Adjusted cumulative odds ratios for progressive loss of alveolar bone of between 2.2 for well-controlled diabetics and up to more than 11 for patients with poor glycemic control have been reported for subjects suffering from type 2 DM [1]. About one third of DM cases are undiagnosed [2]. Considering the very high prevalence of type 2 DM in Kuwait of about $15 \%$ [3], a remarkably high amount of periodontitis may actually be accounted for by DM in this country. Although certain microorganisms in dental plaque are considered to be the main cause of destructive periodontitis, ignoring established risk factors like smoking or DM would most probably lead to therapeutic failure.

Glucometers are commonly used by diabetic patients for home monitoring of blood glucose levels. Recently, more sensitive self-monitoring devices have been devel-

\section{KARGER \\ Fax +4161306 1234 E-Mail karger@karger.ch} www.karger.com
(C) 2004 S. Karger AG, Basel $1011-7571 / 04 / 0136-0361 \$ 21.00 / 0$

Accessible online at:

www.karger.com/mpp
Hans-Peter Müller

Department of Surgical Sciences

Faculty of Dentistry, Kuwait University

PO Box 24923, 13110 Safat (Kuwait)

Tel. +965 2664502, ext. 7133, Fax +965 2634247, E-Mail hp.muller@hsc.kuniv.edu.kw 
oped for testing small amounts ( $<2 \mu \mathrm{l})$ of blood obtained from areas much less sensitive than fingertips, such as the forearm, upper arm, thigh, or base of thumb. In general, accuracy of these novel glucometers has been acceptable [4]. An even more sensitive device, the Freestyle ${ }^{\circledR}$ Blood Glucose Monitoring System, needs only $0.3 \mu$ of blood [5] and may actually allow for totally painless testing of blood oozing from the gingival crevices of patients with mild or moderate gingivitis during routine periodontal examination. This might be of considerable interest to the dental practitioner since this glucometer, if sufficiently accurate, could be a simple and relatively inexpensive inoffice screening device for any patient suspected to have diabetes, or a way to monitor blood sugar levels in known diabetics. In previous studies, in-office screening of gingival blood glucose level in known diabetics [6] and periodontitis patients [7] has been evaluated. However, these devices have required considerably larger blood volumes than those that usually ooze from the crevice after probing in mild or moderate gingivitis. Blood from the gingival crevice after probing is generally a mixture of capillary blood and gingival crevice fluid, an inflammatory exudate [8]. In particular in healthy situations glucose levels in gingival crevice fluid are considerably lower than blood glucose levels [9]. Thus, the question may arise whether glucose levels measured in blood samples obtained during periodontal examination are sufficiently related to glucose levels measured in capillary fingerstick blood (CFB).

The aim of this study was to investigate the feasibility of measuring glucose levels in capillary blood obtained during routine periodontal probing in patients with various degrees of gingival inflammation using the new and very sensitive FreeStyle ${ }^{\circledR}$ self-monitoring glucometer.

\section{Material and Methods}

The study population was recruited among incoming patients of Kuwait University Dental School, Kuwait and Babhani Clinic, Salmiya, Kuwait. Exclusion criteria for screening for DM were: (1) any indication for antibiotic prophylaxis; (2) any bleeding disorder; (3) any medication interfering with the coagulation system, or (4) severe systemic disease such as cardiovascular, renal, hepatic, or immunological disorders. Informed consent was obtained. The 46 patients ( 20 male, 26 female) of the present study were between 12 and 56 years of age, mean $( \pm$ SD) $36 \pm 12$ years. Seven patients (15\%) knew they had diabetes; 3 had type 1 and 4 type 2 DM.

Periodontal conditions were assessed at 6 sites of every tooth present. Periodontal probing depth and clinical attachment level were measured with a calibrated probe (PCPKU13B, Hu Friedy, Germany) to the next millimeter. Bleeding on probing was assessed
Table 1. Periodontal conditions of 46 patients: Blood glucose in CFB and GCB samples

\begin{tabular}{lcc}
\hline & Range & Mean (SD) \\
\hline Age & $12-56$ & $36(12)$ \\
Sites & $96-192$ & $167(18)$ \\
Sites bleeding on probing & $4-162$ & $53(37)$ \\
Sites with periodontal probing & & \\
$\quad$ depth of $\geq 4 \mathrm{~mm}$ & $0-101$ & $15(21)$ \\
CFB glucose, $\mathrm{mmol} / \mathrm{l}(\mathrm{n}=45)$ & $1.39-11.50$ & $5.72(2.06)$ \\
GCB glucose, $\mathrm{mmol} / \mathrm{l}(\mathrm{n}=31)$ & $1.17-10.00$ & $4.33(2.11)$ \\
\hline
\end{tabular}

during $20 \mathrm{~s}$ after probing. A site with more profuse bleeding was chosen for collecting the gingival crevice blood (GCB) sample. The area was isolated with cotton rolls to prevent saliva contamination and dried with compressed air. Probing was repeated until a sufficient amount of blood appeared in the gingival crevice. The FreeStyle Blood Glucose Monitoring System (TheraSense Inc., Alameda, Calif., USA) was used according to manufacturer's recommendations. Immediately before measuring glucose levels in $\mathrm{GCB}$, a CFB sample was drawn from the right index finger using a disposable sterile lancet. Seventeen patients gave consent for another sample from the left index finger to test the reliability of the glucometer device. The agreement between the two methods was studied according to Bland and Altman [10]. It was assumed that differences were found with $95 \%$ confidence within limits defined as mean difference \pm 1.96 times the standard deviation of differences (coefficient of agreement). Student's and paired t tests were employed, as appropriate. Multiple linear regression analysis was applied in order to search for clinical covariates potentially influencing the difference of glucometer readings.

\section{Results}

Plaque-induced gingivitis was diagnosed in 24 patients. Twenty-two patients presented with at least 1 site with a periodontal probing depth of $\geq 5 \mathrm{~mm}$ in combination with loss of clinical attachment of $\geq 2 \mathrm{~mm}$ and were diagnosed as periodontitis patients. Clinical periodontal conditions are shown in table 1 . The paired CFB samples revealed a mean difference of 0.18 and limits of agreement of $\pm 1.38 \mathrm{mmol} / \mathrm{l}$. GCB samples revealed glucometer readings in only 32 cases (range $1.17-10.00 \mathrm{mmol} / \mathrm{l}$ ), whereas an error message was obtained in 14 cases (30\%) because of too small sample volume. Forty-five CFB readings ranged between 1.39 and $11.50 \mathrm{mmol} / \mathrm{l}$. In 1 case with uncontrolled type $1 \mathrm{DM}$ an error message was obtained. The mean difference between GCB and CFB readings was $-1.22 \mathrm{mmol} / \mathrm{l}$. As can be seen in figure 1 , $95 \%$ of the differences could be found between -4.12 and $+1.67 \mathrm{mmol} / \mathrm{l}$ (coefficient of agreement $2.89 \mathrm{mmol} / \mathrm{l}$ ). 
Table 2. Multiple linear regression analysis

\begin{tabular}{lrll}
\hline & Estimate & SE & $\mathrm{p}$ \\
\hline Constant & 0.194 & 0.749 & 0.798 \\
Glucose in CFB sample, mmol/1 & -0.231 & 0.156 & 0.149 \\
Sites bleeding on probing & -0.000 & 0.011 & 0.987 \\
$\begin{array}{l}\text { Sites with periodontal probing depth } \\
\quad \text { of } \geq 4 \mathrm{~mm}\end{array}$ & -0.006 & 0.015 & 0.700 \\
\hline
\end{tabular}

Dependent variable: difference between GCB and CFB glucose concentration $(\mathrm{mmol} / \mathrm{l}) . \mathrm{R}^{2}=0.052, \mathrm{p}=0.226$.

There was no tendency for the variation to change with the magnitude of measurements. Spearman's rank correlation coefficient was insignificant at 0.101 , pointing to no proportional error. If low amounts of GCB prohibited a glucometer reading, the number of sites bleeding on probing was significantly lower ( 26 vs. $67, p<0.001)$, as was the number of periodontal probing depths of $\geq 4 \mathrm{~mm}$ ( 2 vs. $21, p<0.01$ ). Although GCB readings could be obtained especially in subjects with elevated numbers of sites bleeding on probing and increased probing depths, these covariates, if considered in a multiple regression model, did not influence the validity of the measurement (table 2).

\section{Discussion}

The current classification of periodontal diseases and conditions [11] lists DM-associated gingivitis under dental plaque-induced gingival diseases modified by systemic factors. It is a consistent feature found in children with poorly controlled type $1 \mathrm{DM}$. In these cases, the level of diabetic control is a more important aspect than plaque control in relation to the severity of gingival inflammation [12]. Moreover, periodontitis has been proposed as the 6th complication of DM [13]. In fact, there is a twoway relationship between DM and periodontitis. On the one hand, poorly controlled DM increases the risk for developing destructive periodontitis and impairs treatment outcome. On the other, chronic inflammatory periodontal disease considerably complicates diabetic control [14]. It had been estimated that about one third of type $2 \mathrm{DM}$ cases are undiagnosed, and screening for undiagnosed type $2 \mathrm{DM}$ is highly recommended [15]. There are compelling reasons to screen for DM especially among patients seeking dental treatment.

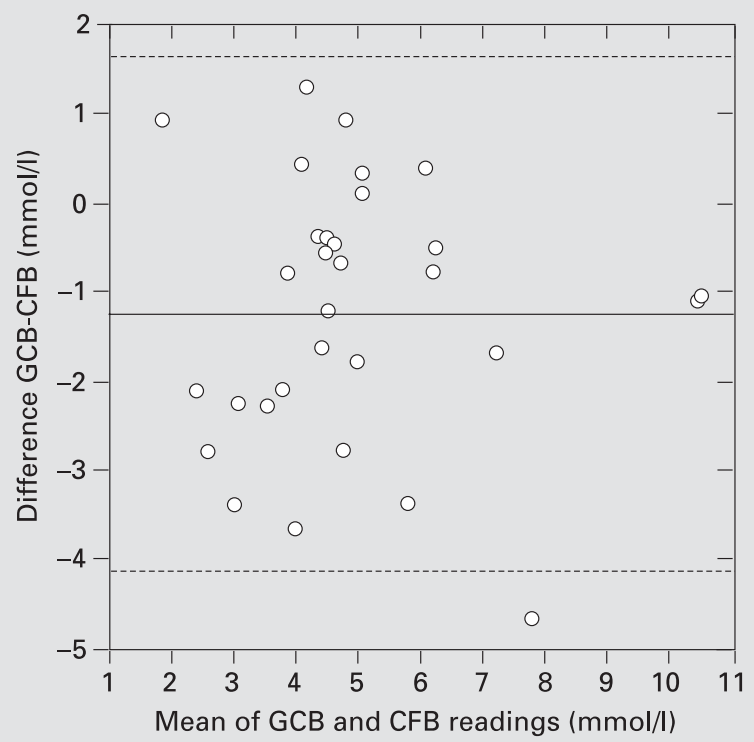

Fig. 1. Bland-Altman plot of differences between glucose levels $(\mathrm{mg} / \mathrm{dl})$ in GCB and CFB on means of both measurements. The dotted lines represent the limits of agreement. The mean difference was $-1.22 \mathrm{mmol} / \mathrm{l}$. Spearman's rank correlation coefficient $\mathrm{r}=$ 0.101 (NS).

In two recent studies gingival blood obtained during routine periodontal procedures was used for glucose determination [6, 7]. In the study by Parker et al. [6] gingival blood samples of 10-15 $\mu$ l were collected from 50 diabetic patients with unknown periodontal status. The authors mention that in some cases calculus was removed to help facilitate collection of a sufficient volume of blood with modified plastic pipettes. Excellent correlations between glucose measurements in gingival blood and the corrected laboratory standard of $r=0.975$ as well as a low mean prediction error were reported. Although the authors claimed feasibility of GCB for in-office blood glucose testing, a large volume of 10-15 $\mu \mathrm{l}$ can usually not be collected after simple probing one or several periodontal sites. It is self-evident that possible advantages of measuring glucose levels directly in GCB are lack of any pain, and easy and safe collection of the sample. Beikler et al. [7] used a self-monitoring device in 45 periodontitis patients, which requires only a volume of $3 \mu$ l. They reported a high correlation $(r>0.98)$ between glucose levels in fingerstick blood and blood sampled during standard periodontal probing, and concluded that GCB collected

Med Princ Pract 2004;13:361-365 
during diagnostic periodontal examination may be an excellent source of blood for glucometric analysis. According to the authors, the technique is safe, easy to perform and comfortable for the patient. It might therefore help to increase the frequency of diabetes screening in the dental office [7]. However, correlation analysis is inappropriate when assessing agreement between two methods of measurement [10]. Furthermore, it was unclear whether a sufficient amount ( $3 \mu \mathrm{l}$ ) of blood can be obtained from the gingival crevice after probing in situations other than severely advanced periodontal disease. The novel FreeStyle ${ }^{\circledR}$ glucometer, which requires only submicroliter volumes of blood and which is one of a few glucometers allowing for direct application of GCB, was therefore employed in the present study to address this question.

Although the present self-monitoring device works with samples of $0.3 \mu \mathrm{l}$ volume, bleeding on probing was not sufficient in every 3 rd case. Compared to glucose levels measured in capillary blood collected after fingerstick, agreement was low. $95 \%$ of the differences were found within limits of between -4.11 and $+1.67 \mathrm{mmol} / \mathrm{l}$. This was not due to unreliability of the device itself, since paired fingerstick samples revealed reasonably narrow limits of repeatability of between -1.22 and $+1.56 \mathrm{mmol} /$ 1. Although GCB readings could be obtained especially in subjects with an elevated number of sites bleeding on probing and increased probing depths, these covariates, if considered in a multiple regression model, did not improve the validity of the measurement. A potential reason for possible discrepancies with former studies may be dilution of blood oozing from the sulcus after probing by gingival crevice fluid. Recently it has been shown that, in fact, higher glucose levels may be detected in gingival crevice fluid at periodontally diseased sites as compared to healthy sites [9]. However, the ratio between blood glucose level and the glucose level in gingival crevice fluid was 2:1 in the former case and 9:1 in the latter. Moreover, although at a considerably lower level, only in dia- betics glucose in gingival crevice fluid correlated with glucose levels in blood [16]. In normal subjects with nonelevated blood glucose levels other factors appear to be responsible for gingival fluid glucose levels [17]. Thus, especially at healthy sites in nondiabetics, gingival crevice fluid containing low levels of glucose may considerably contaminate and dilute the tiny blood sample, leading to profound underestimation of glucose concentration and/or rendering measurements unreliable. The smaller volume of blood used in the present study, the higher proportion of nondiabetics, as well as the large number of subjects with gingivitis might explain some of the deviating results in the studies by Beikler et al. [7], who examined diabetic and nondiabetic patients with moderate or advanced periodontitis, and Parker et al. [6], who examined diabetics with unknown periodontal status.

It has been demonstrated that glucose testing with the FreeStyle ${ }^{\circledR}$ self-monitoring device is sensitive to both the recommended sampling sites and time of sampling. Lee et al. [18] reported small and irrelevant differences between forearm and finger measurements preprandially and $2 \mathrm{~h}$ postprandially. However, the 1-hour postprandial results indicated that patients who test during this period of time could expect to see significant differences between their forearm and finger measurements. The authors concluded that patients who test during this time period should only test using their finger since this would best represent their true glucose level [18]. Especially in hypoglycemic patients, the tip of the finger is still the first choice for sampling [19].

\section{Conclusion}

The present study failed to provide any evidence for the usefulness of gingival crevicular blood for testing blood sugar during routine periodontal examination.

\section{References}

1 Taylor GW, Burt BA, Becker MP, Genco RJ, Shlossman M: Glycemic control and alveolar bone loss progression in type 2 diabetes. Ann Periodontol 1998;3:30-39.

2 Harris MI, Flegal KM, Cowie CC, Eberhardt MS, Goldstein DE, Little RR, Wiedmeyer HM, Byrd-Holt DD: Prevalence of diabetes, impaired fasting glucose, and impaired glucose tolerance in U.S. adults. The Third National Health and Nutrition Examination Survey, 1988-1994. Diabetes Care 1998;21:518-524.
3 Abdella N, Al Arouj M, Al Nakhi A, Al Assoussi A, Moussa M: Non-insulin-dependent diabetes in Kuwait: Prevalence rates and associated risk factors. Diabetes Res Clin Pract 1998;42:187-196.

4 Rheney CC, Kirk JK: Performance of three blood glucose meters. Ann Pharmacother 2000;34:317-321.
5 Feldman B, McGarraugh G, Heller A, Bohannon N, Skyler J, DeLeeuw E, Clarke D: FreeStyle: A small-volume electrochemical glucose sensor for home blood glucose testing. Diabetes Technol Ther 2000;2:221-229.

6 Parker RC, Rapley JW, Isley W, Spencer P, Killoy WJ: Gingival crevicular blood for assessment of blood glucose in diabetic patients. J Periodontol 1993;64:666-672. 
7 Beikler T, Kuczek A, Petersilka G, Flemmig TF: In-dental-office screening for diabetes mellitus using gingival crevicular blood. J Clin Periodontol 2002;29:216-218.

8 Goodson JM: Gingival crevice fluid flow. Periodontol 2000 2003;31:43-54.

9 Ciantar M, Spratt DA, Newman HN, Wilson M: Development of an in vitro microassay for glucose quantification in submicrolitre volumes of biological fluid. J Periodontal Res 2002;37:79-85.

10 Bland JM, Altman DG: Measuring agreement in method comparison studies. Stat Methods Med Res 1999;8:135-160.
11 Armitage GC: Development of a classification system for periodontal diseases and conditions. Ann Periodontol 1999;4:1-6.

12 Mariotti A: Dental plaque-induced gingival diseases. Ann Periodontol 1999;4:7-17.

13 Löe H: Periodontal disease: The sixth complication of diabetes mellitus. Diabetes Care 1993;16:329-334.

14 Grossi SG, Genco RJ: Periodontal disease and diabetes mellitus: A two-way relationship. Ann Periodontol 1998;3:51-61.

15 Harris MI, Eastman RC: Early detection of undiagnosed type 2 diabetes mellitus: A US perspective. Diabetes Metab Res Rev 2000;16: 230-236.
16 Ficara AJ, Levin MP, Grower MF, Kramer GD: A comparison of the glucose and protein content of gingival fluid from diabetics and non-diabetics. J Periodontal Res 1975;10:171175.

17 Hara K, Löe H: Carbohydrate components of the gingival exudate. J Periodontal Res 1969; 4:202-207.

18 Lee DM, Weinert SE, Miller EE: A study of forearm versus finger stick glucose monitoring. Diabetes Technol Ther 2002;4:13-23.

19 McGarraugh G, Price D, Schwartz S, Weinstein R: Physiological influences on off-finger glucose testing. Diabetes Technol Ther 2001; 3:367-376. 